\title{
Influence of sarcopenia focused on critically ill patients
}

\author{
Belgin Akan \\ Department of Anesthesiology and Reanimation, Ankara City Hospital, Health Sciences University, Ankara, Turkey
}

A systemic review was performed to evaluate the epidemiological, pathophysiological, and clinical features of sarcopenia, the relationship of sarcopenia with critical illness and its impact on mortality, and diagnostic methods and treatment modalities. Generally, in the presence of critical illness, sarcopenia is not included in the treatment approach strategies. An intensivist should be aware that sarcopenia may be present in critically ill patients. Although the main modalities against sarcopenia are early mobilization and nutritional support, they can only prevent its development and may have positive effects on prognosis rather than treating the existing sarcopenia.

Key Words: critical illness; intensive care unit; mortality; sarcopenia

\section{INTRODUCTION}

Although sarcopenia was first described by Irwin Rosenberg in 1989 as an age-related decrease in muscle mass ("sarx" or flesh and "penia" or loss) [1], the first definition suitable for clinical use was created by Baumgartner et al. [2] in 1998 as loss of appendicular skeletal muscle mass (ASMM). ASSM is the sum of skeletal muscle mass in the four extremities by two standard deviations or more in healthy young individuals. However, neither Rosenberg [1] nor Baumgartner et al. [2] mentioned muscle strength in these definitions. Finally, the European Working Group on Sarcopenia in Older People (EWGSOP) described sarcopenia in 2010 as undesired loss of skeletal muscle mass and strength and a decrease in physical performance, resulting in increased vulnerability and mortality especially in the elderly [3]. In the pathophysiology-based definition, sarcopenia can be considered a type of organ failure (muscle insufficiency) that can develop mostly chronically (with aging) or infrequently acutely (i.e., during hospitalization or long-term bed rest) [3,4]. In this review, the epidemiological, pathophysiological, and clinical features of sarcopenia are discussed along with the influence of sarcopenia on critically ill patients and their mortality as well as current diagnostic methods and treatment modalities.

\section{EPIDEMIOLOGY OF SARCOPENIA}

ASMM and strength reduction begins in the 4th decade of life. The frequency of sarcopenia in the normal population has been reported by EWGSOP to be 5\%-13\% between the ages of $60-70$ years and $11 \%-50 \%$ above the age of 80 years [3]. With increasing frequency, it is expected to affect approximately 1.2 million people by 2025 and 2 million people by 2050 [5]. Its

\section{Review Article}

Received: September 16, 2020

Revised: November 25, 2020

Accepted: November 25, 2020

Corresponding author

Belgin Akan

Department of Anesthesiology

and Reanimation, Ankara City

Hospital, Health Sciences University,

Üniversiteler Mahallesi, 1604. Cadde,

No: 9, 06800 Çankaya, Ankara,

Turkey

Tel: +90-542-532-51-26

E-mail: belginakan@yahoo.com

Copyright (@) 2021 The Korean Society of Critical Care Medicine

This is an Open Access article distributed under the terms of Creative Attributions Non-Commercial License (https:// creativecommons.org/li-censes/by-nc/4.0/) which permits unrestricted noncommercial use, distribution, and reproduction in any medium, provided the original work is properly cited. 
prevalence is twice as high in men than in women especially in sedentary individuals [6].

With the increase in the aging population, the number of patients hospitalized in the intensive care unit (ICU) has been increasing every year. These patients may be admitted to the ICU for chronic diseases such as heart failure, renal failure, or cancer or for acute conditions such as sepsis, fall injuries, trauma, and postoperative care. There may be decreases in physiological reserves and muscle mass and strength, as well as nutritional deterioration. As there is no practical method that can be performed bedside, it is difficult to evaluate the incidence of sarcopenia in patients in the ICU. In a study using computed tomography (CT) in the ICU, the frequency of sarcopenia was $15 \%-50 \%$ in cancer patients, $30 \%-45 \%$ in patients with liver failure, and $60 \%-70 \%$ in critically ill patients [7].

\section{PATHOPHYSIOLOGY OF SARCOPENIA}

Reduction in innervation and capillary density of skeletal muscle and selective atrophy of type II muscle fibers are possible mechanisms of sarcopenia. In various studies, it has been reported that a relationship exists between sarcopenia and age-related changes in quality, mass, and strength of muscles, as well as metabolic, physiological, and functional disorders that lead to increased morbidity and disability in the elderly [8]. Sarcopenia is thought to be associated with changes in testosterone, estrogen, growth hormone, and angiotensin II levels. Insulin resistance or low insulin level is a common characteristic of diseases that cause extreme muscle wasting. This can promote muscle atrophy due to diminished response to insulin and insulin-like growth factor 1, which enhances muscle protein synthesis and inhibits proteolysis. In addition, in the presence of proinflammatory cytokines (such as tumor necrosis factor $\alpha$ and interleukin-6), muscle breakdown is triggered and causes sarcopenia in the elderly [9].

\section{CLINICAL CHARACTERISTICS OF SARCOPENIA IN THE CRITICALLY ILL PATIENT}

Staging sarcopenia is important in terms of planning the treatment as well as in determining the severity of the disease and predicting its course. There are three stages of sarcopenia. In the "presarcopenia" stage, there is a reduction in muscle mass without change in muscle strength or physical performance. In the "sarcopenia" stage, there is a decrease in muscle strength or physical performance in addition to a decrease in muscle

\section{KEY MESSAGES}

- An intensivist should be aware that sarcopenia can be present in critically ill patients.

- Early mobilization and nutritional support can prevent its development and can have positive effects on prognosis rather than treating existing sarcopenia.

mass. In the presence of "severe sarcopenia," muscle mass, muscle strength, and physical performance are decreased [3]. Knowing the stages of sarcopenia can help determine treatment modalities and recovery goals [10].

Sarcopenia as classified by EWGSOP is either primary or secondary. Primary sarcopenia is only associated with advancing age, with no other reason. Secondary sarcopenia is related to other causes like non-use of muscles, inflammatory disease, malnutrition, and malignancy as well as chronic diseases like cardiac, pulmonary, or renal diseases. In many elderly people, the etiology of sarcopenia is multifactorial, and its classification as primary or secondary might not be possible [3]. EWGSOP classified sarcopenia as acute or chronic in its 2019 version. Acute sarcopenia develops rapidly in the last 6 months of hospitalized elderly patients and is usually associated with an acute illness or injury. Chronic sarcopenia, which takes more than 6 months to develop, is associated with chronic and progressive conditions [11].

Patients are admitted to the ICU with various diagnoses. Patients with sarcopenia are particularly vulnerable in the presence of major physiologic stressors including trauma, major surgery, and critical illness. Due to advanced age and presence of chronic comorbidities, some patients may already have sarcopenia when admitted to ICU [12]. In the presence of primary or secondary sarcopenia, the response to sepsis may be impaired, and mortality has been shown to be higher in patients with sepsis [13]. Patients requiring surgery are often admitted to the ICU. In addition to comorbidities, stress factors related to major surgical intervention negatively affect the short- and long-term prognoses in sarcopenic patients. All these are factors that can prolong ICU and hospital stays [1417]. Likewise, major postoperative complications and the consequent length and cost of hospital stay are five times higher in major surgeries in sarcopenic patients [12].

In the ICU, patients should be stabilized quickly, and mechanical ventilation should be ended as soon as possible. $\mathrm{Al}$ though intensive care departments have very successful weaning protocols, weaning difficulties are $10 \%-30 \%$ higher compared to those in the ward [17]. The pathophysiology of 
weaning failure is often multifactorial, including comorbidities with dysfunction of the lung, heart, and diaphragm/respiratory muscles. Thus, weaning failure is expected to be high in sarcopenic patients since sarcopenia impairs the function of the diaphragm/respiratory muscles [18]. Once connected to a ventilator, sarcopenic patients show higher mortality than non-sarcopenic patients [19-22]. Recently, reports are increasing on high mortality in patients with low skeletal muscle mass when they arrive at the ICU $[21,22]$.

\section{DIAGNOSIS}

EWGSOP reported that decrease in muscle mass and function must be exhibited for diagnosis of sarcopenia. Muscle function can be measured through several physical performance tests and use of specific devices. Difficulties in cooperation and mobilization of these tools can make it difficult to diagnose sarcopenia in the ICU. EWGSOP reported that reduction in muscle mass can be detected through the total or appendicular skeletal muscle mass index (SMMI) [23].

Baumgartner et al. [2] suggested using dual-energy X-ray absorptiometry to measure muscle mass and using the ratio of muscle mass to patient height squared $\left(\mathrm{kg} / \mathrm{m}^{2}\right)$ to measure lean body mass, amount of adipose tissue, and bone mineral density separately and noninvasively. However, the main disadvantages of this method involve the effect of hydration state of the patient and its two-dimensional properties [24]. Lean body mass can be measured through the bioelectrical impedance method. Due to its noninvasive features, this method is easy to apply. However, it cannot measure muscle mass directly and instead measures the electrical transmission of the entire body based on muscle mass. This method is affected by the body's fluid balance, standardization of which can be difficult in the ICU [25].

$\mathrm{CT}$ and magnetic resonance imaging (MRI) methods are considered the gold standards for evaluation of muscle mass [25]. However, there are limitations due to high cost and difficulty in application especially in the presence of critical illness. With these methods, lean muscle mass, adipose tissue, and even fat infiltrations in the muscle can be evaluated. Both CT and MRI use cross-sectional measurement of body compositions. The user performs the measurements after marking fat or muscle components in the software $[25,26]$. In this technique, the subcutaneous fat/muscle ratio and visceral/subcutaneous fat tissue ratio are used. Cross-sectional analysis with $\mathrm{CT}$ at the L3 vertebral level correlate with all body tissue measurements. In this method, the total cross-sectional area in- cluding adipose tissue, psoas, paraspinal muscles (erector spinae, quadratus lumborum), and abdominal wall muscles (transversus abdominis, external and internal oblique, rectus abdominus) is evaluated [27]. Due to the difference in body composition in men and women in abdominal CT, the sexspecific SMMI threshold value is $52.4 \mathrm{~cm}^{2} / \mathrm{m}^{2}$ in men and 38.5 $\mathrm{cm}^{2} / \mathrm{m}^{2}$ in women [28].

When performing bedside evaluation in critical patients, assessment of skeletal muscle by ultrasound (USG) can be useful [26]. However, it has yet to be included in the diagnostic algorithm due to uncertainties such as type of USG probe (linear or convex), anatomical region to be measured (abdomen, lower, or upper extremity), and patient position during examination (supine, prone, or standing). Additionally, it has difficulties such as maintaining immobility of the patient, standardizing the pressure to be applied with the probe, and determining how the probe will be inclined [29].

\section{PROGNOSIS}

The ICU population is very heterogeneous; a large proportion of patients is of advanced age and suffering from chronic comorbidities such as cancer and cardiovascular or renal failure. These comorbidities and presence of sarcopenia are an effective predictor of weaning difficulty, prolonged hospital and ICU stay, and increased mortality and morbidity [30]. The presence of sarcopenia during hospitalization in the ICU contributes to sepsis, resistant infections, neuropathy, and ventilator-associated pneumonia. It is important to reveal the cause of sarcopenia in terms of treatment planning. It remains unknown whether the close relationship between sarcopenia and increased mortality arises from the sarcopenia itself or the muscle destruction resulting from concomitant disease $[31,32]$. In the current pandemic, acute sarcopenia can coexist with coronavirus disease 2019 (COVID-19) and might have a negative effect on duration of mechanical ventilation and prognosis [33].

\section{DIFFERENTIAL DIAGNOSIS}

It is especially important to distinguish sarcopenia from cachexia, a metabolic syndrome characterized by loss of muscle mass with or without loss of fat mass due to underlying disease. Similar mechanisms, such as mitochondrial dysfunction, insulin resistance, changes in protein metabolism, and the presence of inflammation play a role in both sarcopenia and cachexia. Cachexia differs from sarcopenia primarily 
through loss of body weight [34]. In sarcopenia, loss of muscle mass can be accompanied by morphologic traits such as normal weight, overweight, or even obesity and fat infiltration [6]. Cachexia is a multi-organ syndrome with underlying diseases such as cancer, chronic infection, chronic obstructive pulmonary disease, and chronic heart failure. It is characterized by a decrease in body weight, destruction of muscle and adipose tissue, and often anorexia. Most cachectic patients can be sarcopenic, but not all sarcopenic patients are cachectic [35]. This shows that the physical appearance of the patient can be misleading when predicting sarcopenia.

Frailty and sarcopenia are clinical syndromes that can occur with general weakness due to aging. Frailty can be defined as a reduction in the ability to react physically and psychosocially to stress. The importance of early diagnosis of frailty in the elderly is increasing. Early diagnosis is important to protect patients from negative consequences such as falling, hospitalization, and death. Most frail older people may be sarcopenic, and some older people with sarcopenia also are frail [35].

\section{TREATMENT}

Awareness of sarcopenia has recently increased. Since it is related to age, preventive efforts should be performed for the target community. Insufficient energy intake, limitation of physical activity due to prolonged bed rest, and accompanying depression are additional risk factors for sarcopenia and functional decline in hospitalized individuals. In coexistence of sarcopenia with critical illness, there is currently no systematic treatment protocol. However, the following measures and practices have been found to be useful in reducing the severity of disease.

\section{Exercise}

By stimulating protein synthesis in muscles with exercise, muscle mass formation increases and allows faster adaptation to the effort. Early mobilization in the ICU prevents destruction of muscles and increases quality of life by creating a positive effect on the mood of the patient in the recovery process [36]. The number of skeletal muscle fibers can be increased with resistive exercises like weight lifting and stretching [37]. Sarcopenia is a reversible condition when diagnosed early. For patients hospitalized in the ICU, exercise and mobilization as early as possible are critical. In cases of inability to exercise, passive exercise can be used. Although there is not enough knowledge on their long-term effects, nutrition and exercise have a strong additive or synergistic effect with different methods of action in sarcopenic patients [38].

\section{Nutritional Support}

Since nutrition has an important role in the pathophysiology of sarcopenia, it is important in prevention and treatment of sarcopenia. In middle age, cellular and molecular changes reduce the response to nutrition and physical activity, resulting in greater muscle breakdown [38]. Consumption of food decreases by $25 \%$ among older people, and the quality of the food eaten is impaired. Malnutrition plays a role in the pathogenesis of sarcopenia and leads to a decrease in muscle function in many elderly individuals, especially individuals with low body weight $[39,40]$. Malnutrition and significant skeletal muscle mass loss arise from the low amount of protein intake with aging and increased catabolism due to accompanying chronic diseases. The presence of more than one disease accompanying aging and the use of multiple medications can disrupt nutrition significantly. At the same time, with aging, the response to anabolic stimulation decreases and protein requirement increases compared to those of younger people. Fifty percent or more of the elderly patients admitted to the ICU are malnourished [41].

Nutritional status should be measured with indirect calorimetry in the ICU. In long-term starvation, fat and muscle experience breakdown. Aggressive nutrition protocols can be applied in ICU patients. Dietary proteins provide the amino acids needed for synthesis of muscle proteins and function as an anabolic stimulant. If kidney functions are not impaired, it is recommended that protein be given in high doses in the elderly in the ICU $[41,42]$. Proteins, essential amino acids (EAA), $\beta$-hydroxy $\beta$-methyl butyrate (HMB), vitamin $\mathrm{D}$, calcium, antioxidants, and omega-3 fatty acids are important for skeletal muscle health [43].

Protein provides amino acids required for muscle synthesis. Protein malnutrition impairs immune system function. In addition, it also causes the increased risk of infection, delay in wound healing, increased risk of pressure ulcer formation, and prolonged hospitalization with increased morbidity and mortality [44]. Leucine is an EAA that plays an important role in muscle mass and function by increasing the use of amino acids and proteins in the muscles. There is evidence that leucine can activate signaling pathways that lead to protein synthesis [45]. When EAA and HMB are given together, they improve muscle mass and function more than protein given alone. There are studies reporting that it is more effective to apply nutritional support with exercise [38]. HMB is a leucine 
metabolite that helps to increase muscle tension and mass and is increasingly used as a nutritional supplement in sarcopenic patients [43]. HMB increases protein synthesis while preventing its breakdown, with increased effect when combined with exercise. When nutritional support is initiated in the ICU, the enteral route is the preferred option. However, in cases where enteral nutrition does not reach the target dose, it is combined with total parenteral nutrition. In critical illness, macro- and micronutrients must be added during feeding. Calorie and protein intake should be $25-35 \mathrm{kcal} / \mathrm{kg} /$ day and $1.2-1.5 \mathrm{~g} / \mathrm{kg} /$ day, respectively, in elderly and critically ill patients hospitalized in the ICU [46-48]. Protein support, especially that enriched with leucine, stimulates protein synthesis in muscle. The greater is the amount of leucine used, the stronger is the protein synthesis enhancing effect. However, with aging, sensitivity to leucine decreases. Whey is the preferred protein in the diet because it is easy to digest and is rich in leucine. The use of EAA enriched with leucine is particularly useful in patients on mechanical ventilators [39].

In recent years, it has been emphasized that vitamin D deficiency is responsible for the pathophysiology of many diseases. It is a common health problem especially among older people. In addition to other benefits, vitamin $\mathrm{D}$ regulates the function and physiology of skeletal muscles. It can stimulate the proliferation and differentiation of skeletal muscle fibers, maintaining and improving muscle strength and physical performance [46-48]. Vitamin D supplements are beneficial for increasing muscle tension. Reduced protein intake and low vitamin $\mathrm{D}$ level have been found to correlate with diminished muscle strength. With aging, serum vitamin D level decreases due to reduced dietary intake, decreased synthesis due to less sunlight exposure, and reduced conversion to 1-25-hydroxy vitamin $\mathrm{D}$ in the kidneys. Serum vitamin D level should be kept at $40 \mathrm{ng} / \mathrm{mL}$ using at least $800 \mathrm{IU} /$ day [48]. When vitamin D was administered with leucine and protein complex, improvement in muscle parameters was shown in elderly sarcopenic patients [39]. Omega-3 fatty acids can prevent age-related loss of muscle mass and improve physical performance by mediating cell signaling function and inflammation-related oxidative damage. Therefore, its use is recommended in elderly critical patients in the ICU $[49,50]$.

\section{Muscle Electrical Stimulation}

It is important to begin physical activity early in the ICU to preserve muscle mass. Neuromuscular electrical stimulation is recommended as an alternative therapy in the early period when conventional physiotherapy and mobilization cannot be performed. If possible, it should be applied together with exercise [51].

\section{CONCLUSION}

An intensivist should be aware that sarcopenia can be present in the critically ill patient. The patient can be admitted to the ICU with sarcopenia or can develop it acutely in the ICU. Although the main modalities against sarcopenia are early mobilization and nutritional support, these only prevent its development and may have positive effects on prognosis rather than treating the existing sarcopenia.

\section{CONFLICT OF INTEREST}

No potential conflict of interest relevant to this article was reported.

\section{ORCID}

Belgin Akan

https://orcid.org/0000-0003-1589-3553

\section{REFERENCES}

1. Rosenberg IH. Summary comments: epidemiological and methodological problem in determining nutritional status of older persons. Am J Clin Nutr 1989;50:1231-3.

2. Baumgartner RN, Koehler KM, Gallagher D, Romero L, Heymsfield SB, Ross RR, et al. Epidemiology of sarcopenia among the elderly in New Mexico. Am J Epidemiol 1998;147:755-63.

3. Cruz-Jentoft AJ, Baeyens JP, Bauer JM, Boirie Y, Cederholm T, Landi F, et al. Sarcopenia: European consensus on definition and diagnosis: report of the European Working Group on Sarcopenia in Older People. Age Ageing 2010;39:412-23.

4. Marzetti E, Calvani R, Tosato M, Cesari M, Di Bari M, Cherubini A, et al. Sarcopenia: an overview. Aging Clin Exp Res 2017; 29:11-7.

5. Santilli V, Bernetti A, Mangone M, Paoloni M. Clinical definition of sarcopenia. Clin Cases Miner Bone Metab 2014;11:17780.

6. Delmonico MJ, Beck DT. The current understanding of sarcopenia: emerging tools and interventional possibilities. Am J Lifestyle Med 2016;11:167-81.

7. Peterson SJ, Braunschweig CA. Prevalence of sarcopenia and associated outcomes in the clinical setting. Nutr Clin Pract 2016;31: 40-8.

8. Marty E, Liu Y, Samuel A, Or O, Lane J. A review of sarcope- 
nia: enhancing awareness of an increasingly prevalent disease. Bone 2017;105:276-86.

9. Han HQ, Zhou X, Mitch WE, Goldberg AL. Myostatin/activin pathway antagonism: molecular basis and therapeutic potential. Int J Biochem Cell Biol 2013;45:2333-47.

10. Liguori I, Russo G, Aran L, Bulli G, Curcio F, Della-Morte D, et al. Sarcopenia: assessment of disease burden and strategies to improve outcomes. Clin Interv Aging 2018;13:913-27.

11. Cruz-Jentoft AJ, Bahat G, Bauer J, Boirie $Y$, Bruyère $O$, Cederholm T, et al. Sarcopenia: revised European consensus on definition and diagnosis. Age Ageing 2019;48:16-31.

12. Friedman J, Lussiez A, Sullivan J, Wang S, Englesbe M. Implications of sarcopenia in major surgery. Nutr Clin Pract 2015;30: 175-9.

13. Shibahashi K, Sugiyama K, Kashiura M, Hamabe Y. Decreasing skeletal muscle as a risk factor for mortality in elderly patients with sepsis: a retrospective cohort study. J Intensive Care 2017;5:8

14. Wang SL, Zhuang CL, Huang DD, Pang WY, Lou N, Chen FF, et al. Sarcopenia adversely impacts postoperative clinical outcomes following gastrectomy in patients with gastric cancer: a prospective study. Ann Surg Oncol 2016;23:556-64.

15. Hanna JS. Sarcopenia and critical illness: a deadly combination in the elderly. JPEN J Parenter Enteral Nutr 2015;39:27381.

16. de Hoogt PA, Reisinger KW, Tegels JJ, Bosmans JW, Tijssen F, Stoot JH. Functional Compromise Cohort Study (FCCS): sarcopenia is a strong predictor of mortality in the intensive care unit. World J Surg 2018;42:1733-41.

17. Pu L, Zhu B, Jiang L, Du B, Zhu X, Li A, et al. Weaning critically ill patients from mechanical ventilation: a prospective cohort study. J Crit Care 2015;30:862.

18. Dres M, Demoule A. Diaphragm dysfunction during weaning from mechanical ventilation: an underestimated phenomenon with clinical implications. Crit Care 2018;22:73.

19. Trethewey SP, Brown N, Gao F, Turner AM. Interventions for the management and prevention of sarcopenia in the critically ill: a systematic review. J Crit Care 2019;50:287-95.

20. Weijs PJ, Looijaard WG, Dekker IM, Stapel SN, Girbes AR, Oudemans-van Straaten HM, et al. Low skeletal muscle area is a risk factor for mortality in mechanically ventilated critically ill patients. Crit Care 2014;18:R12.

21. Moisey LL, Mourtzakis M, Cotton BA, Premji T, Heyland DK, Wade CE, et al. Skeletal muscle predicts ventilator-free days, ICU-free days, and mortality in elderly ICU patients. Crit Care 2013;17:R206.

22. Toptas M, Yalcin M, Akkoc İ, Demir E, Metin C, Savas Y, et al.
The relation between sarcopenia and mortality in patients at intensive care unit. Biomed Res Int 2018;2018:5263208.

23. Cruz-Jentoft AJ, Landi F, Schneider SM, Zúñiga C, Arai H, Boirie $\mathrm{Y}$, et al. Prevalence of and interventions for sarcopenia in ageing adults: a systematic review: report of the International Sarcopenia Initiative (EWGSOP and IWGS). Age Ageing 2014;43:748-59.

24. Laskey MA. Dual-energy X-ray absorptiometry and body composition. Nutrition 1996;12:45-51.

25. Lee K, Shin Y, Huh J, Sung YS, Lee IS, Yoon KH, et al. Recent issues on body composition imaging for sarcopenia evaluation. Korean J Radiol 2019;20:205-17.

26. Rubbieri G, Mossello E, Di Bari M. Techniques for the diagnosis of sarcopenia. Clin Cases Miner Bone Metab 2014;11:1814.

27. van Vugt JL, Levolger S, Gharbharan A, Koek M, Niessen WJ, Burger JW, et al. A comparative study of software programmes for cross-sectional skeletal muscle and adipose tissue measurements on abdominal computed tomography scans of rectal cancer patients. J Cachexia Sarcopenia Muscle 2017;8:28597.

28. Prado CM, Lieffers JR, McCargar LJ, Reiman T, Sawyer MB, Martin L, et al. Prevalence and clinical implications of sarcopenic obesity in patients with solid tumours of the respiratory and gastrointestinal tracts: a population-based study. Lancet Oncol 2008;9:629-35.

29. Stringer HJ, Wilson D. The role of ultrasound as a diagnostic tool for sarcopenia. J Frailty Aging 2018;7:258-61.

30. Kizilarslanoglu MC, Kuyumcu ME, Yesil Y, Halil M. Sarcopenia in critically ill patients. J Anesth 2016;30:884-90.

31. Kou HW, Yeh CH, Tsai HI, Hsu CC, Hsieh YC, Chen WT, et al. Sarcopenia is an effective predictor of difficult-to-wean and mortality among critically ill surgical patients. PLoS One 2019; 14:e0220699.

32. Meyer F, Valentini L. Disease-related malnutrition and sarcopenia as determinants of clinical outcome. Visc Med 2019;35:28291.

33. Morley JE, Kalantar-Zadeh K, Anker SD. COVID-19: a major cause of cachexia and sarcopenia? J Cachexia Sarcopenia Muscle 2020;11:863-5.

34. Argilés JM, Busquets S, Stemmler B, López-Soriano FJ. Cachexia and sarcopenia: mechanisms and potential targets for intervention. Curr Opin Pharmacol 2015;22:100-6.

35. Bauer JM, Sieber CC. Sarcopenia and frailty: a clinician's controversial point of view. Exp Gerontol 2008;43:674-8.

36. Engel HJ, Needham DM, Morris PE, Gropper MA. ICU early mobilization: from recommendation to implementation at three medical centers. Crit Care Med 2013;41(9 Suppl 1):S69- 
80.

37. Zhang L, Hu W, Cai Z, Liu J, Wu J, Deng Y, et al. Early mobilization of critically ill patients in the intensive care unit: a systematic review and meta-analysis. PLoS One 2019;14:e223185.

38. Denison HJ, Cooper C, Sayer AA, Robinson SM. Prevention and optimal management of sarcopenia: a review of combined exercise and nutrition interventions to improve muscle outcomes in older people. Clin Interv Aging 2015;10:859-69.

39. Robinson SM, Reginster JY, Rizzoli R, Shaw SC, Kanis JA, Bautmans I, et al. Does nutrition play a role in the prevention and management of sarcopenia? Clin Nutr 2018;37:1121-32.

40. Calvani R, Miccheli A, Landi F, Bossola M, Cesari M, Leeuwenburgh $\mathrm{C}$, et al. Current nutritional recommendations and novel dietary strategies to manage sarcopenia. J Frailty Aging 2013;2:38-53.

41. Avelino-Silva TJ, Jaluul O. Malnutrition in hospitalized older patients: management strategies to improve patient care and clinical outcomes. Int J Gerontol 2017;11:56-61.

42. Tessier AJ, Chevalier S. An update on protein, leucine, omega-3 fatty acids, and vitamin $\mathrm{D}$ in the prevention and treatment of sarcopenia and functional decline. Nutrients 2018;10:1099.

43. Beaudart C, Rabenda V, Simmons M, Geerinck A, Araujo De Carvalho I, Reginster JY, et al. Effects of protein, essential amino acids, B-hydroxy B-methylbutyrate, creatine, dehydroepiandrosterone and fatty acid supplementation on muscle mass, muscle strength and physical performance in older people aged 60 years and over: a systematic review on the lit- erature. J Nutr Health Aging 2018;22:117-30.

44. Katsanos CS, Kobayashi H, Sheffield-Moore M, Aarsland A, Wolfe RR. A high proportion of leucine is required for optimal stimulation of the rate of muscle protein synthesis by essential amino acids in the elderly. Am J Physiol Endocrinol Metab 2006;291:E381-7.

45. Martínez-Arnau FM, Fonfría-Vivas R, Cauli O. Beneficial effects of leucine supplementation on criteria for sarcopenia: a systematic review. Nutrients 2019;11:2504.

46. Cruz-Jentoft AJ, Dawson Hughes B, Scott D, Sanders KM, Rizzoli R. Nutritional strategies for maintaining muscle mass and strength from middle age to later life: a narrative review. Maturitas 2020;132:57-64.

47. Gropper S, Hunt D, Chapa DW. Sarcopenia and psychosocial variables in patients in intensive care units: the role of nutrition and rehabilitation in prevention and treatment. Crit Care Nurs Clin North Am 2019;31:489-99.

48. Remelli F, Vitali A, Zurlo A, Volpato S. Vitamin D deficiency and sarcopenia in older persons. Nutrients 2019;11:2861.

49. Papadopoulou SK. Sarcopenia: a contemporary health problem among older adult populations. Nutrients 2020;12:1293.

50. Dupont J, Dedeyne L, Dalle S, Koppo K, Gielen E. The role of omega-3 in the prevention and treatment of sarcopenia. Aging Clin Exp Res 2019;31:825-36.

51. Parry SM, Berney S, Granger CL, Koopman R, El-Ansary D, Denehy L. Electrical muscle stimulation in the intensive care setting: a systematic review. Crit Care Med 2013;41:2406-18. 